\title{
A PRELIMINARY LIST OF XYLARIACEOUS FUNGI OF EL CIELO BIOSPHERE RESERVE, IN TAMAULIPAS, MEXICO
}

\author{
Felipe San Martin Gonzalez \\ Biodiversidad Tamaulipeca A.C. \\ Sierra Hermosa \# 617 Fracc. Villa Real \\ 87010 Cd. Victoria, Tamaulipas, México \\ Y \\ JACK D. ROGERS \\ Department of Plant Pathology \\ Washington State Univeristy \\ Pullman, WA 99164-6430, U.S.A.
}

\begin{abstract}
Se enlistan 77 especies y taxa infraespecíficos de hongos xilariáceos colectados en la Reserva de la Biosfera El Cielo. Para cada taxon se indica el tipo de vegetación y el sustrato en que fue encontrado.
\end{abstract}

\section{RESUMEN}

Seventy seven species and infraspecific taxa of xylariaceous fungi collected in El Cielo Biosphere Reserve are listed. For each taxon, the vegetation type and the substrate, are recorded.

El Cielo Biosphere Reserve comprises an area of $144,530.051$ ha. It is located in the southwestern portion of the state of Tamaulipas, in the region known as Sierra de Cucharas or Sierra de Guatemala, in the Sierra Madre Oriental. The reserve is circumscribed by parallels $22^{\circ} 55^{\prime} 30^{\prime \prime}$ and $23^{\circ} 25^{\prime} 50^{\prime \prime} \mathrm{N}$ and meridians $99^{\circ} 05^{\prime} 50^{\prime \prime}$ and $99^{\circ} 26^{\prime} 30^{\prime \prime} \mathrm{W}$ (Anonymous, 1985). Three main vegetation types prevail in the area: medium size subdeciduous tropical forest located between 200 and 800 meters above sea level (masl), cloud forest present between 700 and 1400 masl and oak-pine forest situated between 1400 and 2100 masl (Rzedowski, 1978).

The inventory of vascular plants for the reserve is well-documented (Lof, 1980; Puig \& Bracho, 1987; Johnston et al., 1989), and the record of vertebrates seems to be essentially complete (Miksch \& Sewall, 1942; Harrel, 1951; Hooper, 1953; Martin, 1955a, 1955b, 1958; Alvarez, 1963).

Mycological studies in the reserve are scarce and mostly deal with taxonomic aspects. The best studied groups are the Aphyllophorales (Valenzuela \& Chacón, 1991; Bandala et al., 1993), the Boletales (García, 1993), and dematiaceous Hyphomycetes (Heredia, 1994). 
Xylariaceous fungi are primarily parasites and saprophytes of angiospermous plants, and even the coprophilous representatives of the family Xylariaceae (Pyrenomycetes, Sphaeriales) are nourished primarily by angiospermous remains and are considered to be specialized angiosperm saprophytes (San Martín \& Rogers, 1995a).

The Xylariaceae previously recorded from the reserve are: Hernández et al. (1951) reported one species of Daldinia and one of Xylaria; Heredia (1989) listed Daldinia concentrica (Bolton:Fr.) Ces. \& De Not., D. vernicosa (Schwein.) Ces. \& De Not. = D. fissa C.G. Lloyd, Entonaema liquescens Moell., Xylaria coccophora Mont., X. grammica (Mont.) Fr., X. magnoliae J.D. Rogers, and X. persicaria (Schwein.: Fr.) Berk. \& M.A. Curtis, and San Martín and Rogers (1989, 1993a, 1993b, 1995) described 27 species of Xylaria, four of Biscogniauxia, three of Camillea, two of Kretzschmaria and one of Rosellinia.

In this contribution, the number of xylariaceous fungi known from different vegetation types and substrates of the reserve is expanded to 77 species and 4 varieties, representing $31 \%$ of the total amount of Xylariaceae reported from Mexico up to the present. For each taxon the bibliographic reference of its description is provided.

The specimens of the taxa listed below were collected and identified from 1986 to 1992 and are housed in ITCV.

Table 1. Xylariaceous fungi from El Cielo biosphere reserve in Tamaulipas.

\begin{tabular}{|c|c|c|c|c|c|}
\hline TAXON & SUBSTRATE & MSTF* $^{*}$ & $\mathrm{CF}^{\star \star}$ & $\mathrm{OP}+$ & $\mathrm{AE}++$ \\
\hline $\begin{array}{l}\text { Anthostomella cf. melanosticta } \\
\text { (Ellis \& Everhart, 1887) }\end{array}$ & Bamboo wood & + & - & - & - \\
\hline $\begin{array}{l}\text { Biscogniauxia mediterranea } \\
\text { (San Martín \& Rogers, 1993b) }\end{array}$ & $\begin{array}{l}\text { Wood of unknown } \\
\text { Dicotyledoneae }\end{array}$ & + & - & - & - \\
\hline $\begin{array}{l}\text { Hypoxylon microplacum } \\
\text { (Miller, 1961) }\end{array}$ & $\begin{array}{l}\text { Wood of unknown } \\
\text { Dicotyledoneae }\end{array}$ & + & - & - & - \\
\hline $\begin{array}{l}\text { Biscogniauxia nummularia var. } \\
\text { merrilii (San Martín \& Rogers, } \\
\text { 1993b) }\end{array}$ & $\begin{array}{l}\text { Wood of unknown } \\
\text { Dicotyledoneae }\end{array}$ & + & - & - & - \\
\hline $\begin{array}{l}\text { Biscogniauxia nummularia var. } \\
\text { pseudopachyloma (San Martín } \\
\text { \& Rogers, 1993b) }\end{array}$ & Citrus wood & - & - & - & + \\
\hline $\begin{array}{l}\text { Camillea cyclisca (Laessøe et al., } \\
\text { 1989) }\end{array}$ & $\begin{array}{l}\text { Wood of unknown } \\
\text { Dicotyledoneae }\end{array}$ & - & - & - & + \\
\hline $\begin{array}{l}\text { Camillea magnifica (San Martín } \\
\text { \& Rogers, 1993b) }\end{array}$ & $\begin{array}{l}\text { Guazuma ulmifolia } \\
\text { wood }\end{array}$ & + & - & - & - \\
\hline $\begin{array}{l}\text { Camillea obularia (Rogers et al., } \\
\text { 1991) }\end{array}$ & Mangifera wood & - & - & - & + \\
\hline
\end{tabular}

* Medium size subdeciduous tropical forest; ${ }^{\star \star}$ Cloud forest; + Oak-pine forest; ++ Agro-ecosystem. 
Table 1. Continuation.

\begin{tabular}{|c|c|c|c|c|c|}
\hline TAXON & SUBSTRATE & MSTF* $^{*}$ & $\mathrm{CF}^{\star *}$ & $\mathrm{OP}+$ & $\mathrm{AE}++$ \\
\hline $\begin{array}{l}\text { Camillea punctulata (Laessøe } \\
\text { et al., 1989) }\end{array}$ & $\begin{array}{l}\text { Quercus spp. } \\
\text { wood }\end{array}$ & - & + & + & - \\
\hline $\begin{array}{l}\text { Camillea tinctor Laessøe et al., } \\
\text { 1989) }\end{array}$ & $\begin{array}{l}\text { Populus sp. } \\
\text { and other } \\
\text { Dicotyledoneae }\end{array}$ & + & + & - & - \\
\hline $\begin{array}{l}\text { Creosphaeria sassafras (Ju et al., } \\
\text { 1993) }\end{array}$ & $\begin{array}{l}\text { Wood of unknown } \\
\text { Dicotyledoneae }\end{array}$ & + & - & - & - \\
\hline Daldinia clavata (Ju et al., 1996) & $\begin{array}{l}\text { Wood of unknown } \\
\text { Dicotyledoneae }\end{array}$ & + & - & - & - \\
\hline Daldinia concentrica (Child, 1932) & $\begin{array}{l}\text { Magnolia sp. } \\
\text { and other } \\
\text { Dicotyledoneae }\end{array}$ & - & + & + & - \\
\hline Daldinia grandis (Ju et al., 1996) & Quercus sp. & - & - & + & - \\
\hline $\begin{array}{l}\text { Daldinia eschscholzii (Ju et al., } \\
\text { 1996) }\end{array}$ & $\begin{array}{l}\text { Wood of unknown } \\
\text { Dicotyledoneae }\end{array}$ & + & - & - & + \\
\hline $\begin{array}{l}\text { Entonaema liquescens (Rogers, } \\
\text { 1981) }\end{array}$ & $\begin{array}{l}\text { Wood of unknown } \\
\text { Dicotyledoneae }\end{array}$ & + & - & - & - \\
\hline Entonaema pallida (Martin, 1938) & $\begin{array}{l}\text { Wood of unknown } \\
\text { Dicotyledoneae }\end{array}$ & + & - & - & - \\
\hline $\begin{array}{l}\text { Hypoxylon erythrostoma (Miller, } \\
\text { 1933) }\end{array}$ & $\begin{array}{l}\text { Wood of unknown } \\
\text { Dicotyledoneae }\end{array}$ & - & + & - & - \\
\hline $\begin{array}{l}\text { Hypoxylon haematostroma (San } \\
\text { Martín, 1992) }\end{array}$ & $\begin{array}{l}\text { Wood of unknown } \\
\text { Dicotyledoneae }\end{array}$ & + & - & - & - \\
\hline $\begin{array}{l}\text { Hypoxylon lenormandii (Ju \& } \\
\text { Rogers, 1996) }\end{array}$ & $\begin{array}{l}\text { Wood of unknown } \\
\text { Dicotyledoneae }\end{array}$ & + & - & - & - \\
\hline $\begin{array}{l}\text { Hypoxylon shearii (Ju \& Rogers, } \\
\text { 1996) }\end{array}$ & Quercus wood & - & + & + & - \\
\hline $\begin{array}{l}\text { Hypoxylon subgilvum (Ju \& Ro- } \\
\text { gers, 1996) }\end{array}$ & $\begin{array}{l}\text { Wood of unknown } \\
\text { Dicotyledoneae }\end{array}$ & + & - & - & - \\
\hline $\begin{array}{l}\text { Hypoxylon subrutilum (Ju \& Ro- } \\
\text { gers, 1996) }\end{array}$ & $\begin{array}{l}\text { Wood of unknown } \\
\text { Dicotyledoneae }\end{array}$ & + & - & - & - \\
\hline
\end{tabular}


Table 1. Continuation.

\begin{tabular}{|c|c|c|c|c|c|}
\hline TAXON & SUBSTRATE & MSTF $^{*}$ & $\mathrm{CF}^{\star \star}$ & $\mathrm{OP}+$ & $\mathrm{AE}++$ \\
\hline $\begin{array}{l}\text { Hypoxylon annulatum (Shear, } \\
\text { 1945) }\end{array}$ & Quercus wood & - & + & + & - \\
\hline $\begin{array}{l}\text { Hypoxylon moriforme (Ju \& Ro- } \\
\text { gers, 1996) }\end{array}$ & $\begin{array}{c}\text { Quercus } \\
\text { and other } \\
\text { Dicotyledoneae }\end{array}$ & + & - & + & - \\
\hline $\begin{array}{l}\text { Hypoxylon rosellinioides (Miller, } \\
\text { 1961) }\end{array}$ & $\begin{array}{l}\text { Wood of unknown } \\
\text { Dicotyledoneae }\end{array}$ & + & - & - & - \\
\hline Hypoxylon stygium (Miller, 1961) & $\begin{array}{l}\text { Wood of unknown } \\
\text { Dicotyledoneae }\end{array}$ & + & + & - & - \\
\hline $\begin{array}{l}\text { Hypoxylon thouarsianum (Miller, } \\
\text { 1961) }\end{array}$ & Quercus wood & - & + & + & - \\
\hline $\begin{array}{l}\text { Hypoxylon cf. ticinense (Ju \& } \\
\text { Rogers, 1996) }\end{array}$ & $\begin{array}{l}\text { Crataegus sp. } \\
\text { wood }\end{array}$ & - & - & + & - \\
\hline $\begin{array}{l}\text { Hypoxylon cf. verrucosum } \\
\text { (Theissen, 1909) }\end{array}$ & $\begin{array}{l}\text { Wood of unknown } \\
\text { Dicotyledoneae }\end{array}$ & + & + & - & - \\
\hline $\begin{array}{l}\text { Kretzschmaria sp. aff. cetrarioides } \\
\text { (San Martín y Rogers, 1993a) }\end{array}$ & $\begin{array}{l}\text { Wood of unknown } \\
\text { Dicotyledoneae }\end{array}$ & + & - & - & - \\
\hline $\begin{array}{l}\text { Kretzschmaria sp. aff. heliscus } \\
\text { (San Martín \& Rogers, 1993a) }\end{array}$ & $\begin{array}{l}\text { Wood of unknown } \\
\text { Dicotyledoneae }\end{array}$ & + & + & - & - \\
\hline $\begin{array}{l}\text { Kretzschmariella culmorum (Ju \& } \\
\text { Rogers, 1994) }\end{array}$ & Bamboo wood & + & - & - & - \\
\hline $\begin{array}{l}\text { Nemania bipapillata (Pouzar, } \\
\text { 1995) }\end{array}$ & $\begin{array}{l}\text { Wood of unknown } \\
\text { Dicotyledoneae }\end{array}$ & + & - & - & - \\
\hline $\begin{array}{l}\text { Nemania confluens (Laessøe \& } \\
\text { Spooner, 1994) }\end{array}$ & $\begin{array}{l}\text { Wood of unknown } \\
\text { Dicotyledoneae }\end{array}$ & + & - & - & - \\
\hline Nemania effusa (Pouzar, 1985) & $\begin{array}{l}\text { Wood of unknown } \\
\text { Dicotyledoneae }\end{array}$ & - & + & - & - \\
\hline $\begin{array}{l}\text { Nemania subannulalata (Van der } \\
\text { Gucht, 1995) }\end{array}$ & $\begin{array}{l}\text { Wood of unknown } \\
\text { Dicotyledoneae }\end{array}$ & + & - & - & - \\
\hline Poronia oedipus (Dennis, 1957) & Cow dung & - & - & - & + \\
\hline $\begin{array}{l}\text { Rosellinia evansii (Laessøe \& } \\
\text { Spooner, 1994) }\end{array}$ & $\begin{array}{l}\text { Wood of unknown } \\
\text { Dicotyledoneae }\end{array}$ & - & - & + & - \\
\hline
\end{tabular}


Table 1. Continuation.

\begin{tabular}{|c|c|c|c|c|c|}
\hline TAXON & SUBSTRATE & MSTF* $^{*}$ & $\mathrm{CF}^{\star \star *}$ & $\mathrm{OP}+$ & $\mathrm{AE}++$ \\
\hline $\begin{array}{l}\text { Rosellinia sublimbata (San Martín } \\
\text { \& Rogers, 1995b) }\end{array}$ & $\begin{array}{l}\text { Wood of unknown } \\
\text { Monocotyledoneae }\end{array}$ & + & - & - & - \\
\hline $\begin{array}{l}\text { Ustulina deusta (San Martín, } \\
\text { 1992) }\end{array}$ & $\begin{array}{l}\text { Wood of unknown } \\
\text { Dicotyledoneae }\end{array}$ & + & + & - & - \\
\hline $\begin{array}{l}\text { Xylaria adscendens (San Martín } \\
\text { \& Rogers, 1989) }\end{array}$ & $\begin{array}{l}\text { Quercus and other } \\
\text { Dicotyledoneae }\end{array}$ & - & + & - & - \\
\hline $\begin{array}{l}\text { Xylaria alata (San Martín \& Ro- } \\
\text { gers, 1989) }\end{array}$ & $\begin{array}{l}\text { Buried Dicotyle- } \\
\text { doneae remains }\end{array}$ & + & - & - & - \\
\hline $\begin{array}{l}\text { Xylaria amphithele (San Martín } \\
\text { \& Rogers, 1989) }\end{array}$ & Fallen leaves & + & - & - & - \\
\hline $\begin{array}{l}\text { Xylaria anisopleura (San Martín } \\
\text { \& Rogers, 1989) }\end{array}$ & $\begin{array}{l}\text { Wood of unknown } \\
\text { Dicotyledoneae }\end{array}$ & + & + & - & - \\
\hline $\begin{array}{l}\text { Xylaria brachiata (San Martín } \\
\text { \& Rogers, 1989) }\end{array}$ & $\begin{array}{l}\text { Wood of unknown } \\
\text { Dicotyledoneae }\end{array}$ & - & + & - & - \\
\hline $\begin{array}{l}\text { Xylaria coccophora (San Martín } \\
\text { \& Rogers, 1989) }\end{array}$ & $\begin{array}{l}\text { Wood of unknown } \\
\text { Dicotyledoneae }\end{array}$ & + & + & - & - \\
\hline $\begin{array}{l}\text { Xylaria cordovensis (Cooke, } \\
\text { 1883) }\end{array}$ & $\begin{array}{l}\text { Wood of unknown } \\
\text { Dicotyledoneae }\end{array}$ & - & + & - & - \\
\hline Xylaria cubensis (Dennis, 1956) & $\begin{array}{l}\text { Wood of unknown } \\
\text { Dicotyledoneae }\end{array}$ & + & + & - & - \\
\hline $\begin{array}{l}\text { Xylaria curta (Callan \& Rogers, } \\
\text { 1990) }\end{array}$ & $\begin{array}{l}\text { Wood of unknown } \\
\text { Dicotyledoneae }\end{array}$ & - & + & - & - \\
\hline Xylaria enteroleuca (Miller, 1934) & $\begin{array}{l}\text { Wood of unknown } \\
\text { Dicotyledoneae }\end{array}$ & + & + & - & - \\
\hline $\begin{array}{l}\text { Xylaria feejeensis (San Martín, } \\
\text { 1992) }\end{array}$ & $\begin{array}{l}\text { Wood of unknown } \\
\text { Dicotyledoneae }\end{array}$ & + & - & - & - \\
\hline $\begin{array}{l}\text { Xylaria frustulosa (Jong \& Ro- } \\
\text { gers, 1970) }\end{array}$ & $\begin{array}{l}\text { Wood of unknown } \\
\text { Dicotyledoneae }\end{array}$ & + & - & - & - \\
\hline $\begin{array}{l}\text { Xylaria gracillima (San Martín, } \\
\text { 1992) }\end{array}$ & $\begin{array}{l}\text { Wood of unknown } \\
\text { Dicotyledoneae }\end{array}$ & + & - & - & - \\
\hline Xylaria grammica (Dennis, 1956) & $\begin{array}{l}\text { Wood of unknown } \\
\text { Dicotyledoneae }\end{array}$ & + & - & - & - \\
\hline
\end{tabular}


Table 1. Continuation.

\begin{tabular}{|c|c|c|c|c|c|}
\hline TAXON & SUBSTRATE & MSTF* & $\mathrm{CF}^{\star \star}$ & $\mathrm{OP}+$ & $\mathrm{AE}++$ \\
\hline $\begin{array}{l}\text { Xylaria guazumae (San Martín \& } \\
\text { Rogers, 1989) }\end{array}$ & $\begin{array}{l}\text { Guazuma ulmifolia } \\
\text { fruits }\end{array}$ & + & - & - & - \\
\hline $\begin{array}{l}\text { Xylaria cf. holmbergi (Saccardo, } \\
\text { 1882) }\end{array}$ & $\begin{array}{l}\text { Wood of unknown } \\
\text { Dicotyledoneae }\end{array}$ & + & - & - & - \\
\hline $\begin{array}{l}\text { Xylaria ianthinovelutina (Dennis, } \\
\text { 1956) }\end{array}$ & $\begin{array}{l}\text { Fruit (mainly } \\
\text { pods) remains }\end{array}$ & + & - & - & - \\
\hline $\begin{array}{l}\text { Xylaria juniperus var. asperula } \\
\text { (Starback, 1901) }\end{array}$ & $\begin{array}{l}\text { Wood of unknown } \\
\text { Dicotyledoneae }\end{array}$ & - & + & - & - \\
\hline $\begin{array}{l}\text { Xylaria kegeliana (San Martín \& } \\
\text { Rogers, 1989) }\end{array}$ & $\begin{array}{l}\text { Wood of unknown } \\
\text { Dicotyledoneae }\end{array}$ & + & - & - & - \\
\hline $\begin{array}{l}\text { Xylaria longiana (San Martín \& } \\
\text { Rogers, 1989) }\end{array}$ & Quercus wood & - & + & + & - \\
\hline Xylaria longipes (Rogers, 1983) & Acer wood & - & + & - & - \\
\hline Xylaria magnoliae (Rogers, 1979) & $\begin{array}{l}\text { Magnolia fruit } \\
\text { remains }\end{array}$ & - & + & - & - \\
\hline $\begin{array}{l}\text { Xylaria aff. mellisii var. nuda (San } \\
\text { Martín \& Rogers, 1989) }\end{array}$ & $\begin{array}{l}\text { Wood of unknown } \\
\text { Dicotyledoneae }\end{array}$ & + & - & - & - \\
\hline Xylaria multiplex (Dennis, 1956) & $\begin{array}{l}\text { Wood of unknown } \\
\text { Dicotyledoneae }\end{array}$ & + & - & - & - \\
\hline $\begin{array}{l}\text { Xylaria musooriensis (Dargan, } \\
\text { 1982) }\end{array}$ & $\begin{array}{l}\text { Wood of unknown } \\
\text { Dicotyledoneae }\end{array}$ & + & - & - & - \\
\hline $\begin{array}{l}\text { Xylaria oxyacanthae (San Martín } \\
\text { \& Rogers, 1989) }\end{array}$ & Fruit remains & + & - & - & - \\
\hline $\begin{array}{l}\text { Xylaria persicaria (San Martín \& } \\
\text { Rogers, 1989) }\end{array}$ & $\begin{array}{l}\text { Liquidambar sp. } \\
\text { fruits }\end{array}$ & - & + & - & - \\
\hline $\begin{array}{l}\text { Xylaria phosphorea (Dennis, } \\
\text { 1956) }\end{array}$ & $\begin{array}{l}\text { Wood of unknown } \\
\text { Dicotyledoneae }\end{array}$ & - & + & - & - \\
\hline $\begin{array}{l}\text { Xylaria aff. piperiformis (San } \\
\text { Martín, 1992) }\end{array}$ & Soil & + & - & - & - \\
\hline $\begin{array}{l}\text { Xylaria poitei (Rogers \& Callan, } \\
\text { 1986a) }\end{array}$ & $\begin{array}{l}\text { Wood of unknown } \\
\text { Dicotyledoneae }\end{array}$ & + & - & - & - \\
\hline
\end{tabular}


Table 1. Continuation.

\begin{tabular}{|c|c|c|c|c|c|}
\hline TAXON & SUBSTRATE & MSTF* $^{*}$ & $\mathrm{CF}^{\star \star *}$ & $\mathrm{OP}+$ & $\mathrm{AE}++$ \\
\hline $\begin{array}{l}\text { Xylaria polymorpha (Rogers \& } \\
\text { Callan, 1986b) }\end{array}$ & $\begin{array}{l}\text { Wood of unknown } \\
\text { Dicotyledoneae }\end{array}$ & + & - & - & - \\
\hline $\begin{array}{l}\text { Xylaria scruposa (San Martín \& } \\
\text { Rogers, 1989) }\end{array}$ & $\begin{array}{l}\text { Wood of unknown } \\
\text { Dicotyledoneae }\end{array}$ & + & + & - & - \\
\hline $\begin{array}{l}\text { Xylaria telfairii (San Martín \& } \\
\text { Rogers, 1989) }\end{array}$ & $\begin{array}{l}\text { Wood of unknown } \\
\text { Dicotyledoneae }\end{array}$ & - & + & - & - \\
\hline $\begin{array}{l}\text { Xylaria uniapiculata (San Martín } \\
\text { \& Rogers, 1989) }\end{array}$ & $\begin{array}{l}\text { Wood of unknown } \\
\text { Dicotyledoneae }\end{array}$ & + & - & - & - \\
\hline
\end{tabular}

\section{ACKNOWLEDGEMENTS}

The senior author likes to thank the Consejo Nacional de Ciencia y Tecnología and the Consejo del Sistema Nacional de Educación Tecnológica de México, for providing him grants to conduct field and laboratory work in the reserve. He likewise thanks the brilliant mycologist, Dr. Yu-Ming Ju for his valuable aid in the identification of the specimens of Hypoxylon. Moreover, San Martín thanks Biól. Lucrecia García Alanís, Biól. Arnulfo Moreno, Dr. Efrén Cázares, Biól. Bertha Baldazo, Dr. Pablo Lavín, Biól. Catalina Flores, M.C. Gonzalo Guevara, M.C. Jesús García, and Biól. María Concepción Herrera for their aid in collecting and the latter also for her aid in identifying plant material.

Jack D. Rogers thanks the National Science Foundation for providing him with a grant to conduct laboratory work.

Both authors express their recognition to two anonymous reviewers who improved the manuscript.

\section{LITERATURE CITED}

Alvarez, T. 1963. The recent mammals of Tamaulipas, Mexico. Univ. Kansas Publ. Mus. Nat. Hist. 14: 363-473.

Anonymous. 1985. Periódico Oficial del Estado de Tamaulipas. Tomo 110. Núm. 56.

Bandala, V. M., G. Guzmán \& L. Montoya. 1993. Los hongos del grupo de los poliporáceos conocidos en México. Reporte Científico de la Facultad de Ciencias Forestales de la Universidad Autónoma de Nuevo León. No. Esp. 13: 1-55.

Callan, B. E. \& J. D. Rogers. 1990. Teleomorph-anamorph connections and correlations in some Xylaria species. Mycotaxon 36(2): 343-369.

Child, M. 1932. The genus Daldinia. Ann. Missouri Bot. Gard. 19: 429-496.

Cooke, M. C. 1883. On Xylaria and its allies. Grevillea 11: 81-94.

Dargan, J. S. 1982. Xylaria musooriensis: a new species from India. Mycologia 74(3): 523-525. 
Dennis, R. W. G. 1956. Some Xylarias of tropical America. Kew Bull. 1956: 401-444.

Dennis, R. W. G. 1957. Further notes on tropical American Xylariaceae. Kew Bull. 1957: 297-332. Ellis, J. B. \& B. M. Everhart. 1887. New species of fungi. Jour. Mycol. 3(4): 41-45.

García, J. 1993. Una lista preliminar de los hongos del suborden Boletineae (Agaricales, Basidiomycetes) en el noreste de México. Reporte Científico de la Facultad de Ciencias Forestales de la Universidad Autónoma de Nuevo León. No. Esp. 13: 116-131.

Harrel, B. E. 1951. The birds of Rancho El Cielo: an ecological investigation in the oak-sweet gum forest of Tamaulipas, Mexico. Master of arts thesis. University of Minnesota, Pilot Knob, Minnesota. 103 pp.

Heredia, G. 1989. Estudio de los hongos de la Reserva de la Biosfera El Cielo, Tamaulipas. Consideraciones sobre la distribución y ecología de algunas especies. Acta Bot. Mex. 7: 1-17.

Heredia, G. 1994. Hifomicetes dematiáceos en bosque mesófilo de montaña. Registros nuevos para México. Acta Bot. Mex. 27: 15-32.

Hernández X., E., H. Crum, W. B. Fox \& A. J. Sharp. 1951. A unique vegetational area in Tamaulipas. Bull. Torr. Bot. Club 78: 458-463.

Hooper, E. 1953. Notes on mammals of Tamaulipas, Mexico. Occas. Pap. Mus. Zool. Univ. Michigan 544: $1-12$.

Johnston, M. C., G. Nixon, G. Nesom \& M. Martínez. 1989. Lista de plantas vasculares conocidas en la Sierra de Guatemala, Gómez Farías, Tamaulipas. Biotam 2: 21-33.

Jong, S. C. \& J. D. Rogers. 1970. Penzigia frustulosa in culture. Mycologia 62: 851-855.

Ju, Y.-M., F. San Martín \& J. D. Rogers. 1993. Three xylariaceous fungi with scolecosporous conidia. Mycotaxon 47: 219-228.

Ju, Y.-M. \& J. D. Rogers. 1994. Kretzschmariella culmorum (Cooke) comb. nov. and notes on some monocot-inhabiting xylariaceous fungi. Mycotaxon 51: 241-255.

Ju, Y.-M. \& J. D. Rogers. 1996. A revision of the genus Hypoxylon. The mycological Society of America. Mycologia Memoir No. 20 and The American Phytopathological Society of America, St. Paul, Minnesota. $365 \mathrm{pp}$.

Ju, Y.-M., J. D. Rogers \& F. San Martín. 1996. A revision of the genus Daldinia. Mycotaxon (in press).

Laessøe, T., J. D. Rogers \& A. J. S. Whalley. 1989. Camillea, Jongiella and light-spored species of Hypoxylon. Myc. Res. 93 (2): 121-155.

Laessøe, T. \& B. M. Spooner. 1994. Rosellinia and Astrocystis (Xylariaceae): new species and generic concepts. Kew Bull. 49: 1-70.

Lof, V. 1980. The ferns of the Rancho del Cielo region. Master of arts thesis. Pan American University, Edinburg, Texas. $161 \mathrm{pp}$.

Martin, G. W. 1938. New or noteworthy fungi from Panama and Colombia II. Mycologia 30: 431-441.

Martin, P. S. 1955a. Herpetological records from the Gómez Farías region of Southwestern Tamaulipas, Mexico. Copeia 3: 173-180.

Martin, P. S. 1955b. Zonal distribution of vertebrates in a Mexican cloud forest. Amer. Nat. 89: 347361.

Martin, P. S. 1958. A biogeography of reptiles and amphibians in the Gómez Farías region, Tamaulipas, Mexico. Misc. Publ. Mus. Zool., Univ. Michigan. 101: 1-102.

Miksch, G. \& O. Sewall. 1942. Birds of the Gómez Farías region, Southwestern Tamaulipas. Journ. of Ornithology 59: 1-35.

Miller, J. H. 1933. Some new species of Hypoxylon. Mycologia 25: 321-328.

Miller, J. H. 1934. Xylariaceae. In: Chardon, C. E. \& R. A. Toro (eds.). Mycological explorations of Venezuela. Monographs of Univ. of Puerto Rico Series B, No. 2. Rio Piedras, Puerto Rico. pp. 195-220.

Miller, J. H. 1961. A monograph of the world species of Hypoxylon. Univ. Georgia Press. Athens. 158 pp.

Petrini, L. E. \& E. Müller. 1986. Haupt- und Nebenfruchtformen europäischer Hypoxylon: Arten (Xylariaceae, Sphaeriales) und verwandter Pilze. Mycologia Helv. 1: 501-627. 
San Martín y Rogers: Preliminary List of Xylariaceae Fungi of El Cielo Biosphere Reserve

Pouzar, Z. 1985. Reassessment of the Hypoxylon serpens-complex II. Ces. Mykol. 39: 129-134.

Puig, H. \& R. Bracho. 1987. El bosque mesófilo de montaña de Tamaulipas. Instituto de Ecología A.C. México D.F. 189 pp.

Rogers, J. D. 1979. Xylaria magnoliae sp. nov. and comments on several other fruit-inhabiting species. Can. J. Bot. 57: 941-945.

Rogers, J. D. 1981. Sarcoxylon and Entonaema (Xylariaceae). Mycologia 73 (1): 28-61.

Rogers, J. D. 1983. Xylaria bulbosa, Xylaria curta, Xylaria longipes in continental United States. Mycologia 75: 457-467.

Rogers, J. D. 1986. Provisional keys to Xylaria species in continental United States. Mycotaxon 26: 85-97.

Rogers J. D. \& B. E. Callan. 1986a. Xylaria poitei: stromata, cultural description, and structure of conidia and ascospores. Mycotaxon 26: 287-298.

Rogers, J. D. \& B. E. Callan. 1986b. Xylaria polymorpha and its allies in continental United States. Mycologia 78: 391-400.

Rogers, J. D., B. E. Callan \& G. J. Samuels. 1987. The Xylariaceae of the rain forests of North Sulawesi. Mycotaxon 29: 113-172.

Rogers, J. D., T. Laessøe \& J. Lodge. 1991. Camillea: new combinations and a new species. Mycologia 83(2): 224-227.

Rogers, J. D., F. San Martín \& Yu-Ming Ju. 1996. Mexican fungi: Xylaria entosulphurea sp. nov. and neotypification of Entonaema globosum. Mycotaxon 58: 483-487.

Rzedowski, J. 1978. La vegetación de México. Ed. LIMUSA. México, D.F. 431 pp.

Saccardo, P. A. 1882. Sylloge fungorum omnium hucusque cognitorum. I Patavii. 768 pp.

San Martín, F. 1992. A mycofloristic and cultural study of the Xylariaceae of Mexico. Ph. D. Thesis. Washington State University, Pullman, Washington. $560 \mathrm{pp}$.

San Martín, F. \& J. D. Rogers. 1989. A preliminary account of Xylaria of Mexico. Mycotaxon 34: 283373.

San Martín, F. \& J. D. Rogers. 1993a. Kretzschmaria, Leprieuria, and Poronia in Mexico. Mycotaxon 48: $179-191$.

San Martín, F. \& J. D. Rogers. 1993b. Biscogniauxia and Camillea in Mexico. Mycotaxon 47: 229258.

San Martín, F. \& J. D. Rogers. 1995a. Notas sobre la historia, relaciones de hospedante y distribución del género Xylaria (Pyrenomycetes, Sphaeriales) en México. Acta Bot. Mex. 30: 21-40.

San Martín, F. \& J. D. Rogers. 1995b. Rosellinia and Thamnomyces in Mexico. Mycotaxon 53: 115127.

Shear, C. L. 1945. Studies of types and authentic specimens of Hypoxylon I. Lloydia 8(4): 245-262.

Starback, K. 1901. Ascomyceten der Ersten Regnellschen Expedition II. Svensk Vet. Akad. Handlingar 27: $1-26$.

Theissen, F. 1908. Novitates riograndenses. Ann. Mycol. 6: 341-352.

Theissen, F. 1909. Xylariaceae Austro-Brasilienses. Ann. Mycol. 7: 141-167.

Valenzuela, R. \& S. Chacón. 1991. Los Poliporáceos de México, III. Algunas especies de la Reserva de la Biosfera El Cielo, Tamaulipas. Rev. Mex. Mic. 7: 39-70.

Van der Gucht, K. 1995. Illustrations and descriptions of Xylariaceous fungi collected in Papua, New Guinea. Bull. Jard. Bot. Nat. Belg. 64: 219-403. 